International Journal of Biomedicine I June 2019 - Volume 9, Issue Suppl_1:

Abstracts From the Second Russian International Conference "Cryo-electron microscopy 2019: achievements and prospects"

POSTER ABSTRACT PRESENTATIONS

SESSION TITLE: NEW METHODS OF SAMPLE PREPARATION AND DATA PROCESSING FOR CRYOELECTRON MICROSCOPY

DOI: 10.21103/IJBM.9.Suppl_1.P41

\title{
Abstract P-41: Cryo-Electron Tomography and 3D Analysis of Detonation Nanodiamonds Hydrosols
}

Alexey A. Mikhutkin ${ }^{1}$, Roman A. Kamyshinsky ${ }^{1,2}$, Nikita M. Kuznetsov ${ }^{1}$, Sergey N. Chvalun ${ }^{1}$, Alexander L. Vasiliev ${ }^{1,2}$

${ }^{1}$ National Research Center "Kurchatov Institute”, Moscow, Russia; ${ }^{2}$ Shubnikov Institute of Crystallography of FSRC “Crystallography and Photonics” RAS, Moscow, Russia

Background: Hydrosols of nanodiamonds prepared from detonation synthesis of carboncontaining explosives find applications in biomedicine, development of novel liquid heat carriers and magnetic liquids. Thus, study of their rheological properties in various liquid media is of great importance. Two unusual rheological features of detonation nanodiamonds (DNDs) hydrosols have been recently revealed: the sharp increase in viscosity and the phase sol-gel transition at a relatively low filler concentration. These features have been explained using the model based on network formation from the chains of faceted DND particles linked together due to the electrostatic interaction of the facets. The 3D spatial distribution, connectivity and agglomeration of the DND particles together with fractal analysis can explain the rheological properties of the hydrosols under the study.

Methods: The specimens were hydrosols of disaggregated DNDs with positive and negative electrokinetic $\zeta$-potential in the concentration range between 1 and $7 \mathrm{wt} \%$. Cryo-Electron Tomography on Titan Krios 60-300 TEM/STEM (FEI, USA) was used for 3D study of DNDs hydrosols at nano- and microscale.

Results: The 3D models of positive and negative $\zeta$-potential DNDs were obtained (example is shown in Fig. 1) and the 3D spatial distribution of DNDs was revealed. It demonstrates the formation of extended fractal structures and chains formed by individual faceted nanodiamond particles. The skeletonization procedure was applied in order to understand the connectivity of objects and percolation. In case of DNDs with positive $\zeta$-potential tomography data demonstrate the formation of a percolation network. However, such network does not form in hydrosols of DNDs with negative $\zeta$-potential. This explains the differences in the rheological behavior at low concentrations of samples with different sign of $\zeta$-potential. The 2D and 3D fractal dimensions were calculated for the mass-fractal and fractal surface. The fractal dimensions, which were estimated from the cryo-electron tomography, are in good correlation with the results of small angle X-ray scattering. 


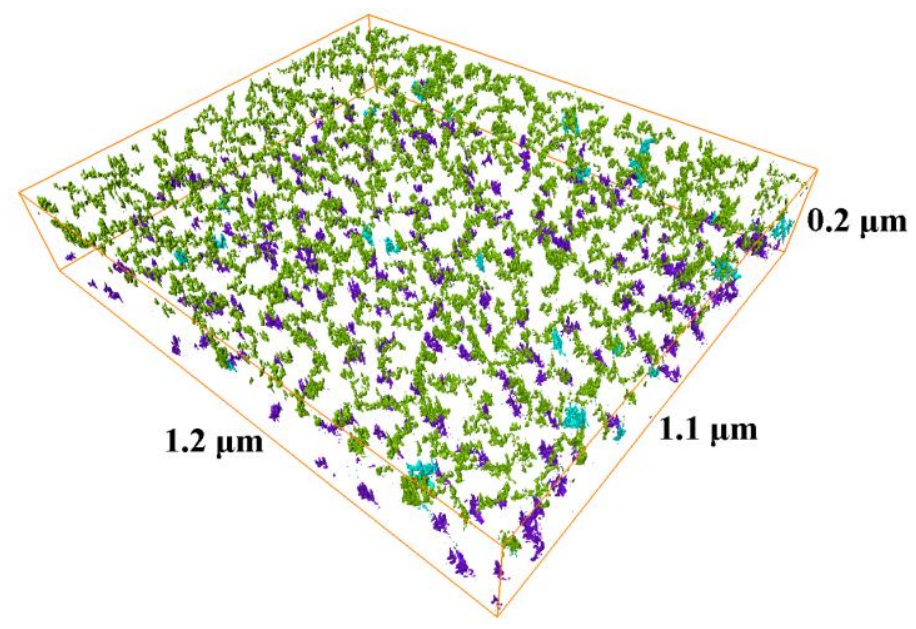

Fig. 1. Cryo-electron tomography $3 D$ model for

1 wt\% nanodiamonds hydrosol with positive $\zeta$-potential.

Conclusion: The results of 3D reconstruction and analysis based on cryo-electron tomography data allow to explain observed features of the rheological behavior associated with DNDs agglomeration and chain formation.

Key Words: 3D reconstruction and analysis $\bullet$ cryo-electron tomography $\bullet$ nanodiamonds hydrosols $\bullet$ fractal analysis

Sources of Funding: The detonation nanodiamonds hydrosols were kindly provided by Prof. A. Ya. Vul' and coworkers from Laboratory Physics for Cluster Structures of Ioffe Institute. This work was partially supported by Russian Foundation for Basic Researches, project 18-29-19117 mk.

International Journal of Biomedicine. 2019;9 Suppl 1: S35. doi: 10.21103/IJBM.9.Suppl_1.P41

(C)2019 International Medical Research and Development Corporation 